\title{
The neuropelveology: from the laparoscopic exposure of the pelvic nerves to a new discipline in medicine?
}

\author{
Marc Possover \\ Received: 20 December 2010/Accepted: 4 February 2011 /Published online: 8 April 2011 \\ (C) Springer-Verlag 2011
}

Keywords Neuropelveology $\cdot$ Pelvic nerves $\cdot$ Sacral plexus $\cdot$ Sciatic nerve $\cdot$ Laparoscopy $\cdot$ Neurosurgery

\section{Background}

The pelvis contains not only different organs such as the bladder, rectum or genital organs, but also pelvic nerves. After the central nervous system and spinal cord, no other part of the body contains so many and such important nerves: pelvic nerves are not only involved in sexuality, voiding and storage of pelvic organs and locomotion, but also in the transport of all sensitive information generated in the lower limbs and pelvis to the central nervous system. Pelvic nerve damages lead therefore to pelvic visceral dysfunctions, problems with locomotion and different kinds of pain. Unfortunately, no specialty deals electively with the pathologies of the pelvic nerves and plexuses!

Pathologies of the pelvic nerves are estimated to be very low in the literature, and most manuals for neurology dedicate them only a few lines; most frequent etiologies reported are then rare neurogenic pathologies and infiltrations by cancers, while iatrogenic damages are estimated to be very rare because of the good protection of the nerves by the pelvic wall. The reality is completely different: neuropathic pain secondary to pelvic surgeries, obstetrical procedures, chemo- and radiotherapies are commonly encountered problems in many medical offices, and

\section{Possover $(\bowtie)$}

Department for Surgical Gynecology \& Neuropelveology,

Hirslanden Clinic,

Witellikerstrasse 40,

CH-8032 Zürich, Switzerland

e-mail: Marc.Possover@hirslanden.ch iatrogenic pelvic dysfunctions are so "common" (especially urinary retention after hysterectomy and incontinence after radical prostatectomy!) that they are widely accepted as a fatality, as the "prize for a good surgery" [1]. This is not surprising if one considers how many invasive procedures in proximity to the pelvic nerves are performed every day all over the world and how many pelvic pathologies do exist which could potentially induce a compression, entrapment or invasion of the pelvic nerves. When surgical nerve injuries occurred, if the lesions are evocated at all, patients are classically sent to neurologists for diagnostic and forensic reasons, are calmed by words and are quite never treated by neurosurgery but by pain killers and antidepressant drugs on the long term.

Incidences of pelvic nerve pathologies are widely underestimated obviously because of lack of awareness that such lesions may exist, lack of diagnosis and acceptance, declaration and report of such lesions. The same phenomenon of "incidence underestimation" is observed with all neurogenic and non-neurogenic pelvic nerve and plexus pathologies.

The most probable reasons for omission of the pelvic nerves in medicine are the complexity of the pelvic nerve system, the difficulties of etiologic diagnosis and-probably the main reason-the limitations of access to the pelvic nerves for neurophysiologic explorations and neurosurgical treatments. Neurosurgical procedures and techniques are well established in nerve lesions of the upper limb, but pelvic retroperitoneal areas and surgeries to the pelvic nerves are still unusual for neurosurgeons. Few opensurgical approaches to the sacral plexus have been described by neurosurgeons for the treatment for traumatic pelvic plexopathies, but these approaches are laborious and invasive, offer only limited access to the different pelvic 
areas and expose patients to the risk of severe vascular complications. Techniques of nerve neuromodulation to control pelvic pain syndromes and dysfunctions are, for the same reasons, limited to the spinal cord and sacral nerve root stimulation that restrict considerably their indications and effectiveness.

\section{Methods}

All these limitations of access to the pelvic nerves and plexuses can now be overcome with laparoscopy: the development of video endoscopy and microsurgical instruments enables a unique access to all pelvic nerves and plexuses, providing the necessary visibility with magnification of the structures and the possibility to work with appropriate instruments [2-4].

\section{Findings}

Using laparoscopic exposure and sparing of the pelvic splanchnic nerves, postoperative functional morbidities can be avoided successfully in radical pelvic surgery [5]. Therefore, iatrogenic dysfunctions such as bladder retention, chronic obstipation, urinary and faecal incontinence or sexual dysfunctions as the "price for an optimal procedure" can be staged as complications and reduced by using selective nerve-sparing techniques.

When nerve injuries had occurred, laparoscopic exploration not only offers an anatomic and functional exploration of the nerves, but also can result in an effective neurosurgical treatment using techniques of nerve(s) decompression or reconstruction the laparoscopic way, too [6]. In non-postsurgical damages, systematic laparoscopic exploration had shown that not only pelvic cancers can induce nerves damages, but also frequent pathologies, such as deeply infiltrating endometriosis, uterine myomas or ovarian processes, or retroperitoneal vascular abnormalities (vascular entrapment), and retroperitoneal fibrosis can also induce pelvic neuropathies that can be treated by laparoscopic nerve decompression/neurolysis [7-10]. Laparoscopy is therefore the essential and logical step in the management of pelvic nerve pathologies that must be indicated as soon as possible, before the nerve damage becomes irreversible and before the process of "pain chronification" starts.

Laparoscopy is also the only technique that enables selective placements of electrodes to all pelvic nerves and plexuses. This technique also called "laparoscopic implantation of neuroprothesis" (abbreviated "LION procedure") permits selective placement of multiple channel electrodes to all pelvic nerves and plexuses that enable multiple strategies of neuromodulation for the control of different pelvic pains and/or dysfunctions at the same time. So, the LION procedure to the sacral plexus (Fig. 1) permits the control of most neuropathic pain syndromes (axonal lesions) [11] and dysfunctions of pelvic organs [12] (chronic pelvic pain, sacral radiculopathies, pudendal/ gluteal/genital pain, urgency syndrome, bladder hyperactivity and/or retention, incontinences...) and of the lower limbs (phantom/residual post-amputation pain, spasticity/ spasms...).

This evolution also presents new therapeutic options in the management of patients suffering from neurogenic pathologies of the peripheral (multiple sclerosis, polyneuropathies, neuromas...) and of the central nervous systems (multiple sclerosis, Parkinson's syndromes, stroke...).

For spinal cord-injured people, since a complete biological cure is unlikely to be developed in the near future, electrical devices are still required to restore functions. The LION procedure enables the implantation of electrodes to the different pelvic nerves involved in pelvic functions and locomotion. In this way, pudendal neuromodulation enables relaxation of the bladder during the filling phase and micturition when desired, setting patients free from catheterization. The sciatic neuromodulation allows for the control of spasticity of the lower extremities by muscle training that constitutes, in combination with the electrically induced skin blood flow improvement, an optimal prophylaxis against decubitus lesions [13, 14]. Blockade of the knees in extension by femoral stimulation with stabilization of the pelvis by concomitant sciatic stimulation enables lower paraplegics (Th7-Th12) to recover an autonomic alternative locomotion. In spina bifida children, the LION procedure offers a unique method for controlling pelvic floor dysfunction using selective pelvic nerve stimulation and bypassing the points of anatomic abnormalities and scar tissue due to previous dorsal surgeries. Laparoscopic neuro-navigation [3] is an essential technique in this

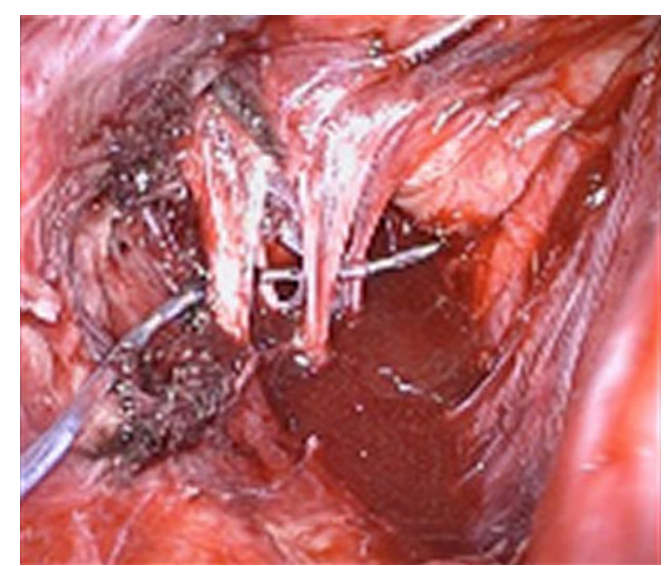

Fig. 1 Sacral plexus LION procedure-octipolar electrode placed to the left sacral nerve roots S2, S3 and S4 
pathology since it grants an exact functional exploration and cartography of the pelvic nerves allowing for a more selective stimulation adapted to specific nerve damages.

\section{Conclusion}

All these new aspects are the results of pioneering work which has been resumed under the term "neuropelveology". This new specialty in medicine focuses on the prevention, diagnosis and treatments of pathologies of the pelvic nerves and plexuses. The dilemma is that all knowledge required for this approach is dispersed into completely different speciality areas, which usually have nothing in common: knowledge in neurology, pelvic neuro-anatomy, pathologies of the pelvic organs and training in laparoscopic (neuro-) surgery are mandatory. Nonetheless, because of the huge number of patients who may profit from these new developments, omission of taking into account the pelvic nerves in medicine is necessary to improve the overall quality. This evolution will require more exchange of ideas between clinical physicians and basic researchers and should encourage young physicians to involve energy and work in fields of clinical and experimental surgeries.

Conflict of interests Prof. Marc Possover has no conflicts of interest or financial ties to disclose.

\section{References}

1. Walker EA, Katon WJ (1991) The prevalence of chronic pain and irritable bowel syndrome in two university clinics. J Psychosom Obstet Gynecol 12:66
2. Possover M (2004) Laparoscopic exposure and electrostimulation of the somatic and autonomous pelvic nerves: a new method for implantation of neuroprothesis in paralysed patients? Gynecol Surg 1:87-90

3. Possover M, Rhiem K, Chiantera V (2004) The "Laparoscopic Neuro-Navigation" - LANN: from a functional cartography of the pelvic autonomous neurosystem to a new field of laparoscopic surgery. Minim Invas Ther Allied Technol 13:362-367

4. Possover M, Chiantera V, Baekerlandt J (2007) Anatomy of the sacral roots and the pelvic splanchnic nerves in women using the LANN technique. Surg Laparosc Endosc Percutan Tech 17:508510

5. Possover M, Quakernack J, Chiantera V (2005) The "LANNtechnique" to reduce the postoperative functional morbidity in laparoscopic radical pelvic surgery. J Am Coll Surg 6:913-917

6. Possover M (2009) Laparoscopic management of neural pelvic pain in women secondary to pelvic surgery. Fertil Steril 91:2720 2725

7. Possover M (2007) Laparoscopic neurolysis of the sacral plexus and the sciatic nerve for extensive endometriosis of the pelvic wall. Minim Invasive Neurosurg 50:33-36

8. Possover M (2009) Laparoscopic management of endopelvic etiologies of pudendal pain in 134 consecutive patients. J Urol $181: 1732-1736$

9. Possover M (2010) New surgical evolutions in management of sacral radiculopathies. Surg Technol Int 19:123-128

10. Possover M, Schneider T, Henle KP (2010) Laparoscopic therapy for endometriosis and vascular entrapment of sacral plexus. Fertil Steril 95:756-758

11. Possover M, Chiantera V, Baekerland J (2007) The laparoscopic approach to control intractable pelvic neuralgia: from laparoscopic pelvic neurosurgery to the LION technique. Clin J Pain 23:821-825

12. Possover M (2010) The laparoscopic implantation of neuroprothesis to the sacral plexus for therapy of neurogenic bladder dysfunctions after failure of percutaneous sacral nerve stimulation. Neuromodulation 13:141-144

13. Possover M (2009) The "sacral LION procedure" for recovery of bladder/rectum/sexual functions in paraplegic patients after explantation of a previous Finetech-Brindley-Controller. J Minim Invasive Gynecol 16:98-101

14. Possover M, Schurch B, Henle KP (2010) New pelvic nerves stimulation strategy for recovery bladder functions and locomotion in complete paraplegics. Neurourol Urodyn 29(8):1433-1438 